\title{
Riesgo de precio en commodities: ¿profundización en la sensibilidad de precios agrícolas ante shocks de tasa de interés?
}

\author{
Price risk in commodities: Sensitivity of agricultural commodities \\ to interest rate shocks?
}

\section{Gonzalo Rondinone* y Esteban Otto Thomasz}

Universidad de Buenos Aires, Argentina

Recibido el 3 de julio de 2015; aceptado el 10 de febrero de 2016

Disponible en Internet el 23 de junio de 2016

\section{Resumen}

El objetivo de este trabajo es someter a prueba el nivel de sensibilidad del precio de commodities agrícolas frente a shocks en la tasa de interés. El estudio se centrará específicamente en el caso del poroto de soja y del maíz, y mediante un sistema de vectores autorregresivos se evaluará la reacción de los precios ante alteraciones en la tasa de interés. En función de los resultados alcanzados, se esbozan algunas implicaciones que puede tener el proceso, sobre todo en relación con el impacto en países dependientes de la exportación de materias primas.

Derechos Reservados (C) 2015 Universidad Nacional Autónoma de México, Facultad de Contaduría y Administración. Este es un artículo de acceso abierto distribuido bajo los términos de la Licencia Creative Commons CC BY-NC-ND 4.0.

Palabras clave: Riesgo de precio; Commodities agrícolas; Tasa de interés; Vectores autrorregresivos Códigos JEL: Q11; G11; C3

\footnotetext{
Abstract

The aim of this study is to test the sensitivity of the price of agricultural commodities against shocks in the interest rate. The study will focus specifically on the case of soybeans and corn, analyzing if in recent years there have been changes in price sensitivity of prices to changes in interest rates. Using a vector

* Autor para correspondencia.

Correo electrónico: gonzalorondinone@economicas.uba.ar (G. Rondinone).

La revisión por pares es responsabilidad de la Universidad Nacional Autónoma de México. 
autoregression model we will test the reaction of prices to changes in interest rates. Depending on the results, we resume some implications of the process, especially in relation to the impact on countries dependent on the export of agricultural commoditites.

All Rights Reserved (C 2015 Universidad Nacional Autónoma de México, Facultad de Contaduría y Administración. This is an open access item distributed under the Creative Commons CC License BY-NC-ND 4.0.

Keywords: Price Risk; Agricultural commodities; Financialization; Autorregressive vector system JEL classification: Q11; G11; C3

\section{Introducción}

A partir del año 2000 se han observado cambios en la dinámica de los precios de commodities, marcando niveles récord de crecimiento de precios y también elevada volatilidad. Asimismo, se ha observado el ingreso de inversores de cartera al mercado de commodities. La inversión de cartera y su influencia en los precios ha sido definida como proceso de financiarización de commodities y ha sido ampliamente estudiada en los últimos años (United Nations, 2009, 2011; Tang y Xiong, 2012).

Esto genera el interrogante de si la entrada de jugadores financieros a dicho mercado ha elevado la reacción de los precios respecto al comportamiento de variables financieras fundamentales como la tasa de interés y el valor del dólar. Existen estudios recientes que analizan la relación entre dichas variables y los precios de commodities (Bastourre, Carrera y Ibarlucia, 2008, Akram, 2009; Curcio, de Jesús, Quirolo y Vilker, 2010; Le Clech, 2013), pero en ningún caso se analiza si la relación se ha profundizado respecto a períodos anteriores.

En este sentido, el objetivo de este trabajo es someter a prueba si se han generado cambios significativos en el nivel de sensibilidad del precio de commodities agrícolas frente a shocks de tasa de interés y de la cotización del dólar durante la última década respecto a un período previo.

En la primera sección de este trabajo se presenta una síntesis del conjunto de relaciones que vinculan la tasa de interés, el valor del dólar y otras variables financieras con el precio de commodities. En la segunda sección se presenta una breve descripción del modelo econométrico utilizado. En la tercera sección se testea mediante un sistema de vectores autorregresivos la sensibilidad del precio del poroto de soja y del maíz ante shocks de tasa de interés y de la cotización del dólar en términos comparativos entre 1990-2003 y 2004-2014. Por último, se esbozan algunas conclusiones en relación con los resultados alcanzados en función de la conveniencia de incorporar commodities a carteras de inversión por un lado, pero sobre todo en relación con el impacto que dicho efecto puede generar en países dependientes de la exportación de materias primas.

\section{Primera sección. Modelización de precios de commodities}

En esta sección se presentan modelos básicos que intentan explicar el comportamiento de los precios de los commodities sobre la base de las relaciones entre la tasa de interés, la cotización del dólar y el nivel de stocks de materias primas. En primer lugar se presentan las ecuaciones básicas que vinculan la tasa de interés y el precio de los commodities para posteriormente presentar los modelos que plantean la reacción de los commodities frente a cambios en la tasa de interés. Seguidamente se presenta la relación entre cotización del dólar y precio de commodities, para culminar con relación entre stocks y precios. El objetivo de la sección es fundamentar la elección de variables que conformarán el modelo que será el núcleo del trabajo. 
Tasa de interés y precio de commodities

La relación entre tasa de interés y commodities tiene larga tradición en la literatura, partiendo de la contribución de Hotelling (1931), quien postula que la tasa de crecimieto de los precios de commodities debería ser análoga a la tasa de interés.

Siguiendo a Akram (2009), una relación básica entre precio de commodities y tasa de interés (bajo el supuesto de mercados eficientes) es la siguiente:

$$
E_{t} p c_{t+1}-p c_{t}=i_{t}+s\left(i_{t}\right)
$$

La ecuación expresa que la revaluación durante un período del precio de un commodity es igual a la tasa de interés más el costo de almacenaje del mismo. Es una práctica usual en la literatura que el costo de almacenaje dependa a su vez de la tasa de interés (Deaton y Laroque, 1996; Yang, Bessler y Leatham, 2001). La relación implica, en mercados eficientes, que el diferencial de precios entre un período y el otro debe ser igual a lo que se hubiese ganado por la venta y colocación a una tasa de interés más el costo de acarreo de un período. Alzas en las tasas de interés reducen la apreciación esperada de commodities.

Otra forma sencilla de entender la relación entre commodities y tasa de interés es la definición explicitada por Hull (2009):

$$
F_{0}=S_{0} e^{(r+u) T}
$$

Donde $F_{0}$ es el precio de un contrato de futuros en el momento presente, $S_{0}$ es el precio spot del activo subyacente del contrato, $r$ es la tasa libre de riesgo, $T$ es el tiempo hasta el delivery del contrato y $U$ es el valor presente de los costos de almacenaje durante la duración del contrato. Reordenando la ecuación se observa que el precio spot es igual al valor descontado de los futuros netos de un costo de almacenaje:

$$
S_{0}=F_{0} e^{-(r+u) T}
$$

Se deduce de la misma que menores tasas de interés elevan el valor presente y, por lo tanto, el precio del commodity.

\section{Reacción de los commodities frente a cambios en la tasa de interés}

Más allá de la relación entre la tasa de interés y el precio de commodities, es relevante analizar la respuesta de estos últimos frente a los cambios en la tasa. En este marco destaca el trabajo de Frankel (1986), basado en el modelo de overshooting de Dornbusch (1976). Para el autor, una contracción monetaria temporaria incrementa la tasa de interés real vía la tasa de interés nominal, a través una caída en la inflación esperada o una combinación de ambas. Esto genera que temporalmente el precio relativo de los commodities caiga más que su valor de largo plazo, hasta el punto de que los mismos sean percibidos como ampliamente subvaluados, generando una expectativa futura de apreciación que es suficiente para contrarrestar la nueva tasa de interés más elevada. En el largo plazo, el nivel de precios se ajusta para cambiar la oferta monetaria real y tanto la tasa de interés real como el precio real de los commodities se retrotraen a los valores iniciales. El supuesto clave que cierra el modelo de overshooting es que los precios de los productos agropecuarios y minerales ajustan rápidamente, mientras que el resto de los precios ajusta lentamente. 
En un trabajo posterior, Frankel (2006) resume que mayores tasas de interés reducen la demanda de aquellos commodities que pueden ser almacenados, e incrementan la oferta de los mismos a través de 3 canales: a) en commodities energéticos como el petróleo, un alza de la tasa de interés incentiva la extracción presente en detrimento de hacerlo en el futuro; b) disminuyendo los incentivos para incrementar inventarios, y c) vía efecto sustitución en aquellos inversores que venden sus posiciones en commodities a otras inversiones financieras como los treasury bills. Contrariamente, una baja de la tasa de interés real tiene el efecto opuesto, bajando los costos de acarrear inventarios e incentivando el efecto sustitución hacia commodities.

\section{Relación entre cotización del dólar y precio de commodities}

Como la mayoría de los commodities cotizan en dólares, una devaluación del dólar incrementa el poder de compra relativo del resto de las monedas mundiales, elevando su precio. Bajo el cumplimiento de la ley de precio único, esto último puede ser interpretado a partir de la siguiente ecuación:

$$
p c^{f}=e+p c
$$

Donde $p c$ es el precio de un commodity en dólares, $e$ es el tipo de cambio nominal del dólar en término de unidades de moneda extranjera y $p c^{f}$ es el precio del commodity valuado en moneda extranjera. Por definición, un commodity es un bien homogéneo y que se transa en los mercados internacionales. Consecuentemente, una depreciación (apreciación) de la moneda de nominación del bien incrementa (disminuye) el poder de compra de los países que son demandantes de ese commodity. De existir algún diferencial, los arbitrajistas, bajo el supuesto de mercados eficientes, se encargarán de que el precio del commodity se incremente (disminuya).

Dornbusch (1985) estudia la relación entre los movimientos en términos reales del dólar y el precio de los mismos. Su conclusión es la misma que se presentó anteriormente, pero agrega que a medida que aumenta la participación de los países demandantes en el total del comercio mundial, estos efectos se amplifican.

Es claro que hay un efecto de simultaneidad entre los 2 factores financieros enumerados. Los movimientos en la tasa de interés y la cotización de la moneda no son independientes. Una baja en la tasa de interés puede conllevar una depreciación del dólar, por lo cual el efecto sobre commodities se da por ambos canales: efecto Fischer vía ecuación de paridad de interés. El análisis de tal efecto está fuera de los alcances de este trabajo.

\section{Relación entre precio, stocks y demanda}

La relación entre la producción y la demanda de commodities juega un rol clave en la determinación de los precios. De acuerdo a Maizels (1994) y Reinhart (1994), las grandes fluctuaciones de precios de commodities pueden ser explicadas por shocks de oferta y demanda relacionada con el commodity físico. La duración de los shocks y la amplitud dependerá de la situación de stocks. Si el impacto puede absorberse a través de stocks, entonces el mismo pierde potencia; en cambio, si los niveles de stocks son bajos, cualquier problema que afecte a la demanda o la oferta tendrá mayor impacto sobre el precio. Parte de los fuertes incrementos de precios del período 2004-2008 y 2009-2013 se dieron coincidentemente con situaciones de muy bajos stocks a nivel mundial para las distintas producciones de commodities agrícolas (principalmente soja, maíz y trigo).

Por otro lado, los cambios producidos a patir de 2000, mayormente en las economías demandantes de commodities, muestran la importancia de focalizarse también en el lado de la demanda, 


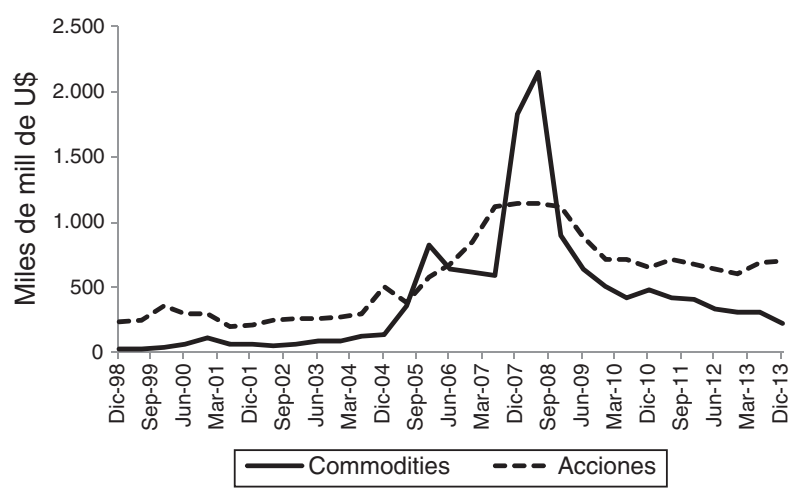

Figura 1. Trading de acciones y derivados OTC.

Fuente: elaboración propia en base a Bank for International Settlements Derivatives Statistics.

particularmente en los llamados «Asian drivers» como movilizadores del ciclo de commodities. Autores como Kaplinsky (2006) y Nissanke (2010) muestran el rol de estos —principalmente China e India — en la demanda de commodities.

\section{Relaciones con otras variables financieras}

Los denominados índices de commodities $^{1}$ han estado a disposición de los inversores desde principio de la década de los noventa, aunque en los últimos años han tenido una fuerte expansión. Estos índices fueron creados para ofrecer al inversor un acceso al mercado de commodities en forma de activo financiero. Durante la década del 2000 muchas firmas lanzaron sus propios índices con distintas formas de construcción para alcanzar determinados objetivos.

En la figura 1 se compara la evolución del trading de acciones y derivados Over-The-Counter (OTC) de commodities. Como puede observarse, el valor de mercado de las posiciones abiertas en commodities se multiplicó por 70 desde el primer dato de la serie hasta el máximo registrado en junio de 2008, período previo al estallido de la crisis internacional. Es interesante notar que durante los primeros años de la década el tamaño del mercado de OTC en acciones fue siempre mayor al de commodities. No obstante, en diciembre de 2004 la serie comienza a mostrar un crecimiento acelerado de las operaciones en OTC relacionadas a commodities que lo llevan, tan solo un año después, a alcanzar el volumen de las posiciones en acciones. Autores como Baker (2014), Basu y Gavin (2010) y Henderson et al. (2012) trabajan sobre dicha línea.

Más allá de la evolución del mercado en sí mismo, un tópico de estudio importante es observar cómo se comportan los retornos de estos índices en relación con otros activos. En otras palabras, ¿los índices se comportan en forma cada vez más similar a otros activos netamente financieros? Tang y Xiong (2012) presentan la rolling correlation de un año tanto para el S\&P 500 como para el S\&P GSCI ${ }^{2}$. Allí puede observase que la correlación se mantuvo en una banda entre 0

\footnotetext{
${ }^{1}$ Son índices que replican el comportamiento de los precios de un conjunto de commodities. La inversión en los mismos se realiza a través de un vehículo deonominado exchangeable trade fund (ETF).

${ }^{2}$ El S\&P GSCI es un índice de commodities ponderados en función de su participación en la producción mundial. El sector energético tiene fuerte participación en el índice. Fue lanzado originalmente en 1991 y es utilizado como benchmark de mercado.
} 
y -0.2 durante la década de los noventa, pero a partir del año 2004 la correlación comienza a incrementarse hasta 0.6, manteniéndose posteriormente en ese nivel. Resulta relevante estudiar este comportamiento en futuras investigaciones, ya que uno de los fundamentos básicos para incorporar commodities a portfolios tradicionales de inversión es su baja correlación con otro tipo de activos financieros. De acuerdo con Tang y Xiong (2012), este incremento en la correlación puede deberse a una mayor inestabilidad macroeconómica a nivel global, pero también a una mayor presencia de inversores financieros en los mercados de commodities.

Es interesante notar que la correlación no solo se incrementó con el S\&P 500, sino que también lo hizo con los mercados emergentes. Comparando la rolling correlation de un año entre el S\&P 500 y el MSCI Emerging Market ${ }^{3}$ se observa lo siguiente: hasta el año 2004 estaba en torno a 0 , a partir de lo cual comienza a subir gradualmente para alcanzar un valor de 0.6 y finalmente estabilizarse en 0.5 (Tang y Xiong, 2012, pp. 35). Este incremento es coincidente con el impacto que las economías emergentes han ganado sobre la economía mundial en los últimos 20 años. En este sentido, Kilian y Park (2009) explican que el rápido crecimiento de China e India son una fuente de explicación de la suba del precio de los commodities. Como muestran Tang y Xiong (2012), la demanda de commodities por parte de las economías emergentes depende positivamente de su crecimiento económico y negativamente del precio del dólar, dado que es la moneda de cambio para este tipo de transacciones. En este caso se observa también una correlación cada vez más negativa entre el retorno del S\&P 500 y el dólar, medido a través del Dollar Index, que mide la fortaleza del dólar ante el euro, el yen, la libra, el franco suizo y la corona sueca. En la misma línea, Silvennoinen y Thorp (2013) estudian las correlaciones de 24 commodities individuales y los principales índices accionarios europeos usando información semanal desde 1990-2009.

Dado que la correlación comienza a incrementarse desde el año 2004, cuando se da un crecimiento fuerte de los índices de commodities, es válido preguntarse si a partir de dicho año se ha profundizado la relación entre precio de commodities y tasa de interés, en vistas de que la tasa es uno de los principales alocadores de activos en el marco de la inversión de cartera.

En síntesis, la relación entre tasa de interés y precios de commodities dista de ser novedosa, partiendo de los fundamentos básicos de la teoría financiera (hipótesis de mercados eficientes, ecuación de paridad de intereses, valuación de derivados) hasta modelos más sofisticados (Dornbusch, Frankel, etc.). En este sentido, el objetivo de este trabajo no es verificar la relación entre tasa de interés y precio de commodities, sino evaluar si dicha relación se ha profundizado debido a nuevos efectos de índole estrictamente financiera. Por ello, en la sección siguiente se presenta un testeo sobre si la relación entre la tasa de interés y el precio de commodities se ha profundizado durante 2004-2014 respecto a 1990-2003.

\section{Segunda sección. Medición de la sensibilidad del precio de la soja y del maíz ante cambios en la tasa de interés}

\section{Modelo de vectores autorregresivos}

El objetivo principal de esta sección es estudiar la respuesta de los precios internacionales de commodities agrícolas ante variaciones en la tasa de interés norteamericana. Si bien las relaciones entre tasa de interés y precio de commodities no son algo reciente (tal como fue descripto en el

\footnotetext{
${ }^{3}$ El MSCI Emerging Market es un índice que busca emular el retorno de los mercados emergentes. Las mayores participaciones individuales son de China, Corea del Sur, Taiwán, Sudáfrica y Brasil.
} 
marco teórico), el crecimiento de la inversión financiera de índices de commodities, entre otros factores, puede haber profundizado dicha relación.

Para estudiar la incidencia de la tasa de interés en el precio de poroto de soja y maíz, se aplica un modelo de vectores autorregresivos (VAR). La elección de esta herramienta de modelización frente a otras alternativas, como las ecuaciones simultáneas, radica en la posibilidad del estudio de funciones de impulso-respuesta. Estas analizan la interacción dinámica que caracteriza al sistema estimado, siendo posible identificarlas con la simulación del modelo propuesto, mostrando la reacción o respuesta de las variables explicadas en el sistema ante cambios en los errores. Un shock en una variable en el período $i$ afectará directamente a la propia variable y, al mismo tiempo, se transmitirá al resto de las variables explicadas a través de la estructura dinámica en el modelo VAR.

Una de las críticas al modelo VAR es que, dada su forma de construcción, la interpretación de los parámetros se vuelve compleja. No obstante, no es el objetivo de este trabajo la estimación de dichos parámetros sino comprender la respuesta de la soja y el maíz ante cambios en la tasa de interés y comprender si esa respuesta ha cambiado en los últimos años.

La técnica aplicada, propuesta por Sims (1980), sugiere que, de existir una verdadera simultaneidad entre un conjunto de las variables, todas deben ser tratadas de la misma manera. No debería existir ex-ante una distinción entre variables endógenas y exógenas. Los modelos de ecuaciones simultáneas que trata la econometría tradicional permiten explicitar esta dependencia mutua entre las variables. Sin embargo, para lograr una formalización adecuada se requiere clasificar las variables en las categorías «endógenas» y «exógenas», distinción que no siempre surge con claridad de la teoría económica subyacente. Sims busca solucionar este problema: en su versión irrestricta trata a todas las variables simétricamente en el sentido de que coloca en el mismo rango a todas las variables del modelo: todas son endógenas, y se especifica cada una de ellas como función lineal de sus propios valores pasados y valores pasados de las restantes variables del sistema.

Estos modelos son utilizados particularmente para analizar la dinámica generada ante el impacto de un shock aleatorio en alguna de las variables del sistema, por lo cual se adapta al objetivo planteado en esta sección.

Con la idea de simplificar la exposición teórica se utiliza un modelo VAR bivariado de primer orden presentando en Brooks (2014).

$$
\begin{aligned}
& Y_{1 t}=\delta_{1}+\phi_{11} Y_{1, t-1}+\phi_{12} Y_{2, t-1}+\varepsilon_{1 t} \\
& Y_{2 t}=\delta_{2}+\phi_{21} Y_{1, t-1}+\phi_{22} Y_{2, t-1}+\varepsilon_{2 t}
\end{aligned}
$$

Puede observarse que cada variable es función lineal de los valores rezagados de ella misma y de las restantes variables incluidas. Cada variable regresando comparte el mismo grupo de variables regresoras. Esto permite interpretar la representación VAR como la forma reducida de un modelo estructural de ecuaciones simultáneas. Para la representación anterior, el modelo estructural correspondiente es:

$$
\begin{aligned}
& Y_{1 t}=b_{10}+b_{12} Y_{2 t}+c_{11} Y_{1, t-1}+c_{12} Y_{2, t-1}+\mu_{1 t} \\
& Y_{2 t}=b_{20}+b_{21} Y_{2 t}+c_{21} Y_{1, t-1}+c_{22} Y_{2, t-1}+\mu_{2 t}
\end{aligned}
$$

Una de las ventajas decisivas, como se mencionó anteriormente, que tiene la modelización VAR frente a otras alternativas es la posibilidad de estudiar funciones de impulso-respuesta sobre las variables. Siguiendo la notación vectorial del modelo se tiene que:

$$
Y_{t}=\delta+\phi_{1} Y_{t-1}+\varepsilon_{t}
$$


Tomando en cuenta la estacionariedad del VAR (1), puede expresarse esto como un vector de medias móviles $(\infty)$ :

$$
\begin{aligned}
& Y_{t}=\delta+\phi_{1} Y_{t-1}+\varepsilon_{t}=\bar{Y}+\sum_{j=0}^{\infty} \phi_{1}^{j} \varepsilon_{t-j} \\
& \bar{Y}=\left[\frac{\bar{Y}_{1}}{\bar{Y}_{2}}\right]
\end{aligned}
$$

Combinando la representación vectorial del modelo estructural con la representación anterior como medias móviles, puede verse que:

$$
\left[\begin{array}{l}
Y_{1 t} \\
Y_{2 t}
\end{array}\right]=\left[\begin{array}{l}
\bar{Y}_{1} \\
\bar{Y}_{2}
\end{array}\right]+\sum_{j=0}^{\infty}\left[\begin{array}{ll}
\phi_{11} & \phi_{12} \\
\phi_{21} & \phi_{22}
\end{array}\right]^{j}\left[\begin{array}{cc}
1 & -b_{12} \\
-b_{21} & 1
\end{array}\right]^{-1}\left[\begin{array}{l}
\mu_{1 t} \\
\mu_{2 t}
\end{array}\right]
$$

Esta representación permite analizar la interacción entre las series. Los coeficientes $\phi_{i k}$ que componen cada una de las matrices pueden utilizarse para generar los efectos de $\mu_{1 t}$ y $\mu_{2 t}$ sobre las trayectorias temporales de $Y_{1 t}$ e $Y_{2 t}$. Estos coeficientes son los multiplicadores del sistema y sus representaciones gráficas constituyen las funciones de impulso-respuesta.

Una cuestión relacionada directamente con la efectividad de las respuestas de los coeficientes de impulso-respuesta es la determinación de los lags óptimos a utilizar en el modelo VAR. El estudio de Ivanov y Kilian (2005) presenta una excelente discusión sobre el criterio óptimo de selección. Se ha demostrado que la dinámica de las respuestas a los impulsos depende críticamente del orden de los lags elegidos que se ajusten a los datos del VAR. Estas diferencias pueden ser lo suficientemente grandes como para afectar la interpretación de las estimaciones. La estrategia más común en los trabajos empíricos es seleccionar un orden para el lag por algún criterio específico y condicionar las estimaciones sobre este a la hora de construir las funciones de impulso-repuesta. Se recomienda utilizar varios criterios de selección para determinar el orden correcto que mejor se ajuste a los datos. Todavía existe una discusión teórica sobre cómo proceder en el caso de que distintos criterios ofrezcan diferentes selecciones de lags. Los autores concluyen que para series mensuales la utilización de Akaike information criterion (AIC) es la adecuada para la selección. Llegan a esta conclusión para muestras de $\mathrm{T}=600$; cuando el $\mathrm{T}$ es menor, por ejemplo 240 , el criterio de AIC es tan adecuado como el Hannan-Quinn information criterion (HQ). A los fines de este trabajo se prioriza en ambos criterios sin perder de vista el resto a los fines de ganar robustez.

Una vez estimado el modelo, puede procederse a excluir algunas variables explicativas en función de su significatividad estadística, pero existen razones que explican que no resulta lo más conveniente. Por un lado, si se mantiene el mismo conjunto de variables explicativas en todas las ecuaciones, entonces la estimación ecuación por ecuación por mínimos cuadrados ordinarios es eficiente. Por otro lado, la presencia de retardos como variables explicativas hace que la colinealidad entre variables explicativas sea importante, lo que hace perder precisión en la estimación del modelo y reduce los valores numéricos de los estadísticos tipo $t$ de Student. Esto hace que no sea una buena estrategia proceder en varias etapas, excluyendo del modelo las variables cuyos coeficientes resultan estadísticamente no significativos, ya que esto puede ser consecuencia de la colinealidad inherente al modelo.

Por otro lado, se espera que las variables analizadas presenten raíces unitarias, por lo cual utilizar un VAR directamente no es el camino adecuado. Este último es un marco general de análisis que muestra las relaciones dinámicas entre variables estacionarias. Por lo tanto, el primer paso será analizar la presencia de raíces unitarias para las variables en niveles. En caso de que 
lo sean, se tomarán las mismas en primeras diferencias, ya que usualmente, si las variables en niveles no son estacionarias, sus primeras diferencias lo serán. De esta forma, un caso particular de modelos VAR es el conocido como vector de corrección de errores (VEC), el cual resulta más indicado para variables que son estacionarias en sus primeras diferencias. El modelo VEC también puede tomar en cuenta relaciones de cointegración entre las variables. Si las variables son estacionarias, en sus diferencias se tiene un modelo con las siguientes características:

$$
\begin{aligned}
& \Delta Y_{1 t}=\delta_{1}+\phi_{11} \Delta Y_{1, t-1}+\phi_{12} \Delta Y_{2, t-1}+\varepsilon_{1 t} \\
& \Delta Y_{2 t}=\delta_{2}+\phi_{21} \Delta Y_{1, t-1}+\phi_{22} \Delta Y_{2, t-1}+\varepsilon_{2 t}
\end{aligned}
$$

Bajo esta especificación puede procederce a estimar con el método de mínimos cuadrados. Introduciendo las relaciones de cointegración entre las variables del mismo orden se tiene el VEC:

$$
\Delta Y_{1 t}=\delta_{1}+\phi_{1} \Delta Y_{2, t}+\phi_{2}\left(Y_{1, t-1}-\gamma Y_{2, t-1}\right)+\mu_{t}
$$

El término $\left(Y_{1, t-1}-\gamma Y_{2, t-1}\right)$ es conocido como término de corrección de error. Dado entonces que $Y_{1, t-1} ; Y_{2, t-1}$ están cointegradas, la combinación en el paréntesis será $I(0)$, aun cuando sus predecesoras no lo eran. Una vez planteada esta corrección, los procedimientos de mínimos cuadrados e inferencia estadística vuelven a ser válidos.

\section{Modelo VAR aplicado}

Respecto al conjunto de commodities estudiados, el estudio se focaliza en el sector agrícola. Específicamente, se ha seleccionado el caso del poroto de soja y del maíz ${ }^{4}$. El testeo gana en robustez al tratar la base de datos de forma mensual, por lo cual se incrementa significativamente la cantidad de observaciones. En la figura 2 se muestran las variables relevantes en niveles.

Tal como se mostró en capítulos anteriores, se parte de los determinantes estudiados por la literatura para especificar el modelo. Se eligen como variables la tasa de interés americana y la cotización del dólar. El precio del poroto de soja y del maíz surgen de los contratos de futuros operados en Chicago, nominados en dólares por tonelada (U\$/tn), y la fuente de información utilizada es el Fondo Monetario Internacional. Para medir la cotización del dólar se utiliza el Dolar Index, el cual representa un índice que mide la fortaleza relativa del dólar ante una canasta de monedas extranjeras (yen, libra esterlina, dólar canadiense, corona sueca y franco suizo). Finalmente, la variable de tasa de interés está representada por la tasa a 10 años de los bonos americanos de madurez constante y la fuente de datos es la Fed de St. Louis.

La metodología aplicada es la siguiente: en primer lugar, la muestra se divide en 2 períodos, 1990-2003 y 2004-2014. La fundamentación de la sección del quiebre de la muestra se basa en que es a partir de 2004 cuando se registra un crecimiento acelerado de las operaciones en OTC relacionadas a commodities, y la correlación entre tales productos y acciones se eleva sustancialmente respecto al período previo, tal como fue descripto en la marco teórico de este trabajo. La segmentación de la muestra permitrá justamente observar si se generan cambios en la respuesta del modelo entre períodos.

\footnotetext{
${ }^{4}$ Se selecciona soja y maíz dado que son cultivos en los que Estados Unidos es el primer productor mundial, teniendo mayor influencia en la fijación de precios internacionales. Además, dentro de Latinoamérica destaca la alta incidencia en la exportación, sobre todo de soja, en los casos de Argentina, Paraguay y, en menor medida, Brasil.
} 

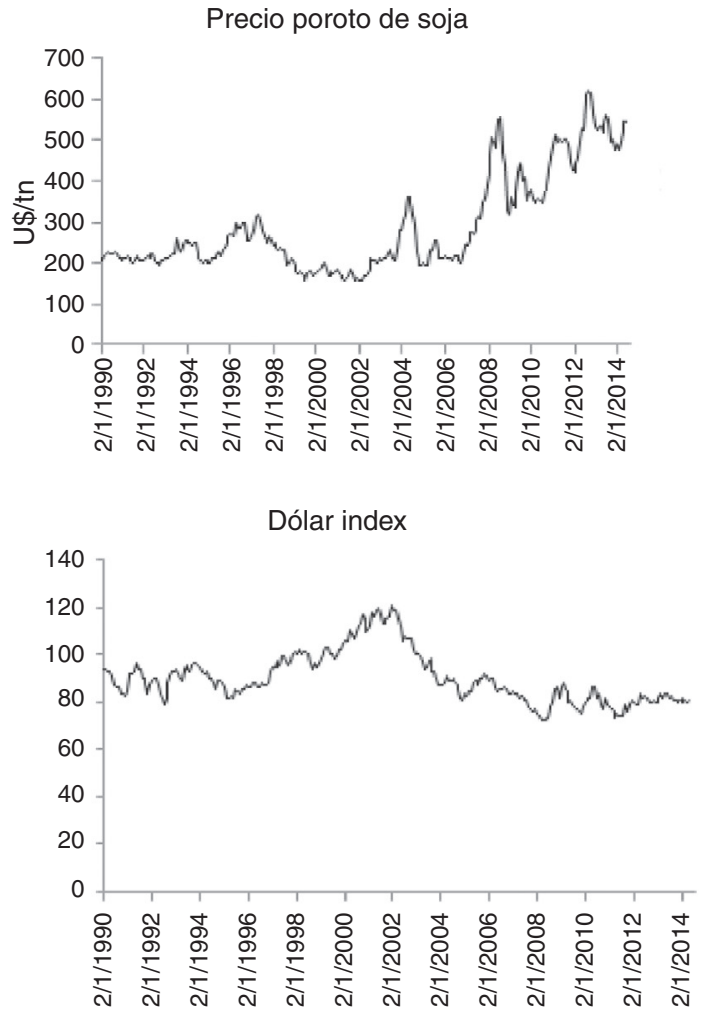

Precio de maíz

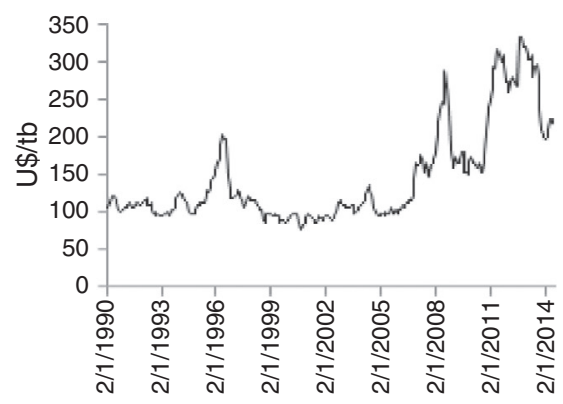

Treasury 10y

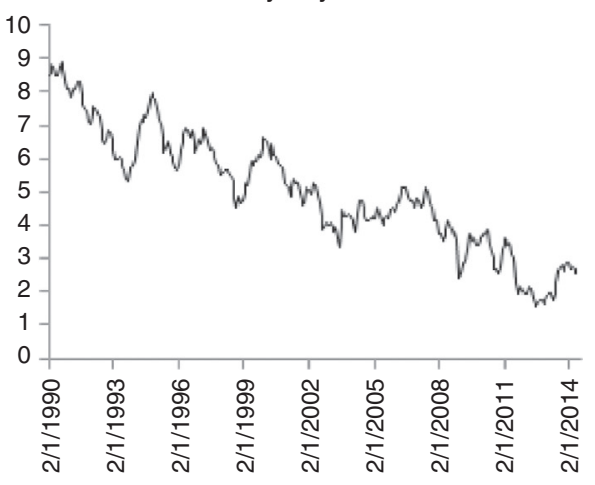

Figura 2. Variables en estudio.

Fuente: elaboración propia en base al FMI y Fed St. Louis.

Al interior de cada período se realizan los siguientes testeos: test de raíz unitaria sobre las variables, a los fines de determinar si se trabajará sobre nivel o diferencias primeras a los fines de ganar estabilidad. Luego, se lleva a cabo el test de cointegración entre variables, para decidir si puede aplicarse un modelo VAR verdaderamente independiente. Luego se realiza un test de estabilidad al modelo VAR propiamente dicho. De ser estable, se estiman las ecuaciones correspondientes; si es inestable, se aplica un modelo VEC. También se define el nivel óptimo de lags. Finalmente, se construyen las ecuaciones de impulso-respuesta introduciendo shocks de tasa equivalente a una desviación estándar.

Como se ha dicho, las variables utilizadas en el análisis tienen una frecuencia mensual y van desde 1990-2014. Fueron computadas con el software Eviews.

Los trabajos de Le Clech (2013), Akram (2009) y Curcio et al. (2010) tratan los determinantes del precio de los commodities y buscan verificar las relaciones teóricas mediante testeos empíricos. Este trabajo parte de una pregunta de investigación distinta: se busca estudiar si la respuesta del precio de commodities agrícolas (soja y maíz) a un shock de tasa de interés ha cambiado su comportamiento durante los períodos de estudio. Para esto se utilizan funciones de impulso-respuesta luego de controlar por distintas variables. A diferencia de los trabajos anteriores, se incluye en el modelo el ratio stock/consumo de ambos productos en Estados Unidos. La correlación con el precio internacional es más alta que si tomamos la producción mundial. Además, extendemos 
el horizonte temporal de análisis hasta el año 2014. En este marco el trabajo es complementario de dicha línea de investigación.

\section{Tercera sección. Resultados del modelo}

A continuación se presentan las gráficas de las ecuaciones de impulso-respuesta, exponiendo el comportamiento simulado mediante el modelo VAR o VEC del precio del poroto de soja y del maíz frente a un shock (positivo) de tasa de interés de magnitud equivalente a un desvío estándar. Como fue expuesto, se divide a la muestra en 2 períodos de idéntica duración de 10 años.

En primer lugar, en las figuras 3 y 4 se observa que la suba de la tasa reduce el precio de ambos commodities. Comparando entre períodos, el efecto es mucho más pronunciado en 2004-2014 también en ambos commodities. Comparando entre commodities, y focalizando en el período 2004-2014, el efecto de la baja del precio es mucho más persistente en el caso del poroto de soja.

Dado que las cotizaciones internacionales de ambos commodities se han incrementado sustancialmente de la primera sección de la muestra a la segunda, se procede a observar la magnitud del shock como proporción del precio promedio. Este control se lo realiza a fines de evitar que la amplificación del impulso en términos absolutos no sea respuesta de mayores precios. Como se observa en la tabla 1 , los shocks representan un porcentaje mayor de la cotización en el período 2004-2014.
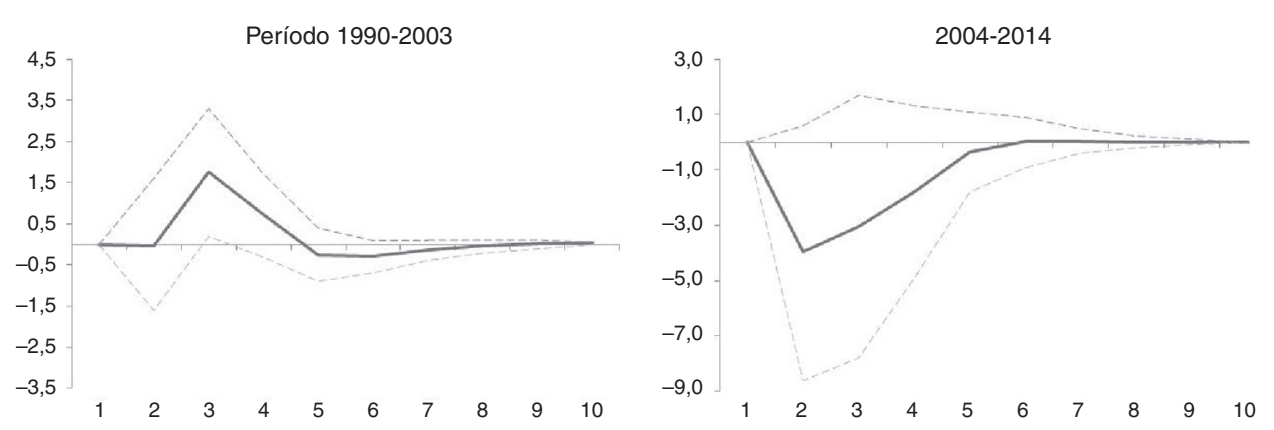

Figura 3. Función de impulso-respuesta: shock de tasa de interés en poroto de soja.
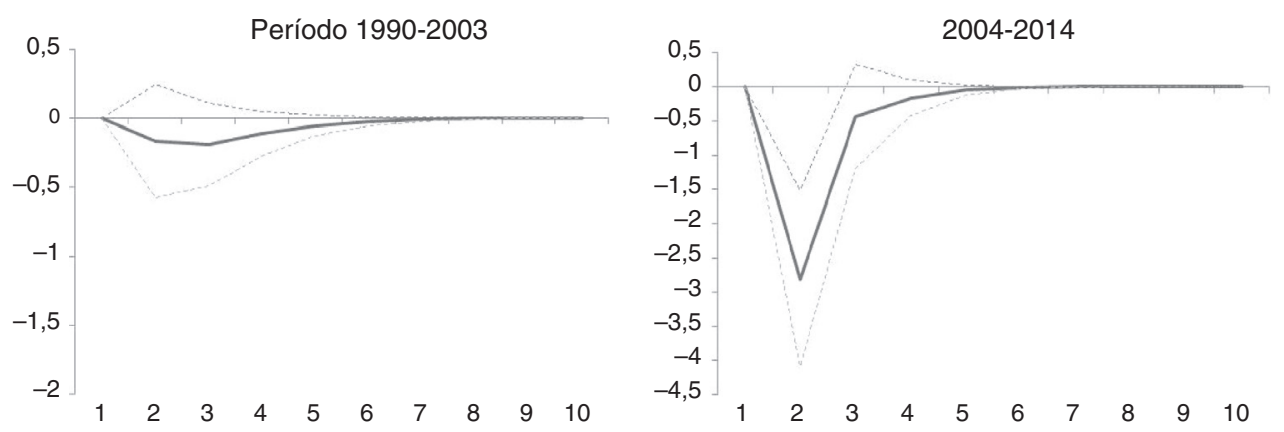

Figura 4. Función de impulso-respuesta: shock de tasa de interés en maíz. 
Tabla 1

Shock de tasa como proporción del precio promedio del período

\begin{tabular}{llr}
\hline & $1990-2003$ & $2004-2014$ \\
\hline Soja & $0.81 \%$ & $1.03 \%$ \\
Maíz & $0.18 \%$ & $1.46 \%$ \\
\hline
\end{tabular}

Fuente: elaboración en propia.
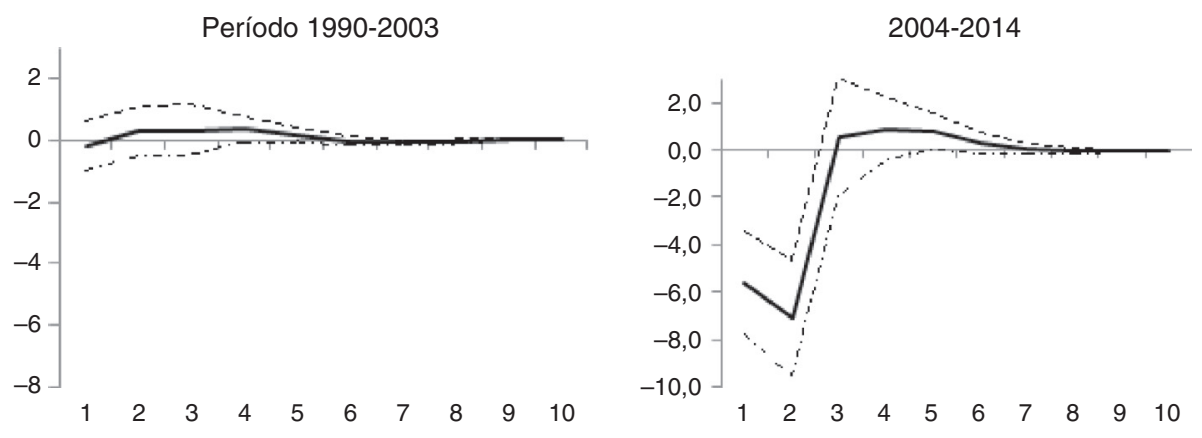

Figura 5. Función de impulso-respuesta: shock de Dolar Index en poroto de soja.

En tanto, tomando la misma división de la muestra se procede al estudio de la función de impulso-respuesta de la soja y el maíz ante un cambio en la variable Dolar Index. En las figuras 5 y 6 puede observarse que un shock de magnitud equivalente a un desvío estándar genera una reducción en los precios, tal como se espera ex-ante. No obstante, durante el período 1990-2003 el efecto se revierte rápidamente en los períodos subsiguientes. En tanto, entre 2004 y 2014 el impacto es mucho más pronunciado y persistente. Este hecho se registra tanto en soja como en maíz, aunque, al igual que con el shock de tasa, en la oleaginosa se amplifica el impacto.

Este resultado reafirma los efectos alcanzados previamente, dadas las relaciones existente entre cotización del dólar, tasas de interés y precios de commodities descriptas en el marco teórico del este trabajo.
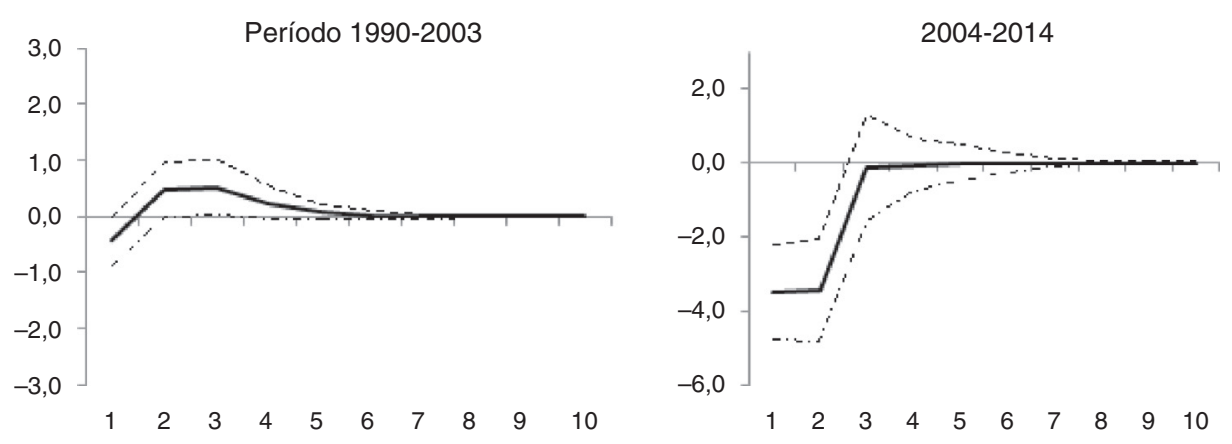

Figura 6. Función de impulso-respuesta: shock de Dolar Index en maíz. 


\section{Conclusiones}

Los resultados de los testeos realizados en este trabajo muestran que en la segunda sección de la muestra correspondiente al período 2004-2014 se observa una profundización de la respuesta del precio del poroto de soja y del maíz frente a cambios en la tasa de interés. Comparando ambos commodities, el resultado es más intensivo en el caso del poroto de soja.

Estos resultados pueden resultar relevantes a la hora de estudiar a los commodities como instrumento de inversión de cartera. Es posible que la amplificación del impacto que fue esbozado en este trabajo, que será ampliado en futuras investigaciones, tenga relación con el comportamiento conjunto de commodities, acciones y bonos, dado el incremento en la correlación entre los mismos. Ello puede generar que se reduzcan los beneficios de la diversificación: determinar si actualmente la incorporación de estos instrumentos a carteras de bonos y acciones optimiza la relación riesgoretorno diversificando riesgos, lo que configura una futura línea de investigación derivada de este trabajo.

Los resultados del trabajo indican que un potencial escenario de suba de tasas de interés internacional puede tener un mayor impacto en el precio que en períodos anteriores. Desde el punto de vista de los países dependientes de las exportaciones de productos agrícolas se podría observar un deterioro de los términos de intercambio y la balanza comercial como se muestra en Massot (2008). En este sentido, en aquellos casos en donde el perfil exportador está definido por commodities agrícolas una suba de tasas podría no solamente afectar a través de la cuenta capital, sino también por una amplificación de la caída de la balanza comercial.

Por otro lado, la lectura opuesta puede hacerse para países consumidores de commodities. Períodos de bajas tasas de interés pueden elevar los precios, ejerciendo presión sobre los precios domésticos de los alimentos. En este caso, el estudio de mecanismos de gestión de riesgos de precios por parte de la gestión pública será una línea de estudio futura.

En síntesis, comprender las dinámicas de los mercados de commodities y los cambios que se suceden en ellos resulta fundamental por las extensas ramificaciones económicas. Impactos desde la inversión de cartera, la determinación del precio de los alimentos, combustible, cuentas externas, fuente de divisas, entre otros, muestran la importancia del estudio de las nuevas tendencias del mercado. 


\section{Anexo 1.}

Tabla A1

Test de raíz unitaria Dickey Fuller aumentado

\begin{tabular}{llc}
\hline Variable/prob & $1990-2003$ & $2004-2014$ \\
\hline Soja & 0.27 & 0.55 \\
D(Soja) & 0.00 & 0.00 \\
Maíz & 0.05 & 0.60 \\
D(Maíz) & 0.00 & 0.00 \\
Tasa 10y & 0.36 & 0.55 \\
D(Tasa 10y) & 0.00 & 0.00 \\
Dolar Index & 0.59 & 0.12 \\
D(Dolar Index) & 0.00 & 0.00 \\
Stock/Cons Soja & 0.13 & 0.29 \\
D(Stock/Cons Soja) & 0.00 & 0.00 \\
Stock/cons Maiz & 0.05 & 0.28 \\
D(Stock/cons Maiz) & 0.00 & 0.00 \\
\hline
\end{tabular}

Fuente: elaboración en propia.

Tabla A2

Test de Selección de lags

\begin{tabular}{lllll}
\hline Criterio & \multicolumn{2}{c}{ 1990-2003 } & & $2004-2014$ \\
\cline { 2 - 5 } & Soja & Maíz & & Soja \\
\hline Final prediction error (FPE) & 2 & 2 & 2 & 1 \\
Akaike information criterion (AIC) & 2 & 2 & 2 & 1 \\
Schwarz information criterion (SC) & 1 & 1 & 1 & 0 \\
Hannan-Quinn information criterion (HQ) & 1 & 1 & 1 & 0 \\
\hline
\end{tabular}

Fuente: elaboración en propia.

Tabla A3

Resumen de resultados de los testeos de estabilidad

\begin{tabular}{llr}
\hline & $1990-2003$ & $2004-2014$ \\
\hline Soja & 8 raíces $<1$ & 8 raíces $<1$ \\
Maíz & 4 raíces $<1$ & 4 raíces $<1$ \\
\hline
\end{tabular}

Fuente: elaboración en propia. 
Tabla A4

Representaciones de las ecuaciones del modelo VAR aplicado

Soja - Período 1990-2003

$\mathrm{D}(\mathrm{SOJA})=0.148 * \mathrm{D}(\operatorname{DOLAR}(-1))-0.068 * \mathrm{D}(\operatorname{DOLAR}(-2))-117.184 * \mathrm{D}(\mathrm{STOCK}(-1))-$

$70.161 * \mathrm{D}(\operatorname{STOCK}(-2))+0.194 * \mathrm{D}(\operatorname{SOJA}(-1))-0.237 * \mathrm{D}(\operatorname{SOJA}(-2))-$

$0.092 * \mathrm{D}(\mathrm{TASA}(-1))+9.321 * \mathrm{D}(\mathrm{TASA}(-2))+0.616$

Maíz - Período 1990-2003

$\mathrm{D}(\mathrm{MAIZ})=0.360 * \mathrm{D}(\mathrm{DOLAR}(-1))+0.081 * \mathrm{D}(\mathrm{DOLAR}(-2))+0.473 * \mathrm{D}(\mathrm{MAIZ}(-1))-$

$0.068 * \mathrm{D}($ MAIZ $(-2))+19.49 * \mathrm{D}(\operatorname{STOCK}(-1))-31.60 * \mathrm{D}(\operatorname{STOCK}(-2))-2.30 * \mathrm{D}(\operatorname{TASA}(-1))+0.98 * \mathrm{D}(\mathrm{TASA}(-2))$

$-0.02$

Soja - Período 2004-2014

$\mathrm{D}(\mathrm{SOJA})=-2.55 * \mathrm{D}(\operatorname{DOLAR}(-1))+1.25 * \mathrm{D}(\operatorname{DOLAR}(-2))+13.52 * \mathrm{D}(\mathrm{STOCK}(-1))-$

$145.68 * \mathrm{D}(\operatorname{STOCK}(-2))+0.291 * \mathrm{D}(\operatorname{SOJA}(-1))+0.030 * \mathrm{D}(\operatorname{SOJA}(-2))-15.41 \mathrm{D}(\operatorname{TASA}(-1))-$

$10.19 * \mathrm{D}(\mathrm{TASA}(-2))+0.522$

Maíz - Período 2004-2014

$\mathrm{D}(\mathrm{MAIZ})=-1.349 * \mathrm{D}(\mathrm{DOLAR}(-1))+0.113 * \mathrm{D}(\mathrm{MAIZ}(-1))-137.203 * \mathrm{D}(\mathrm{STOCK}(-1))-$

$11.519 * \mathrm{D}(\operatorname{TASA}(-1))+0.454$

Fuente: elaboración en propia.

\section{Referencias}

Akram, Q. F. (2009). Commodity prices, interest rates and the dollar. Energy Economics, 31(6), 838-851. http://dx.doi.org/10.1016/j.eneco.2009.05.016

Baker, S. D. (2014). The Financialization of Storable Commodities, Working Paper. In McIntire School of Commerce. Charlottesville, VA: University of Virginia.

Bastourre, D., Carrera, J. y Ibarlucia, J. (2008). Precio de los commodities en Argentina: ¿qué mueve al viento? Ensayos Económicos, 51.

Basu, P. y Gavin, W. T. (2010). What explains the growth in commodity derivatives? Federal Bank of St. Louis Review, 93(1), 37-48.

Borensztein, C. y Reinhart, C. (1994). The Macroeconomic Determinants of Commodity Prices, IMF Working Paper. No. 94/9.

Brooks, C. (2014). Introductory Econometrics for Finance. Cambridge: Cambridge University Press.

Curcio S., de Jesús M., Quirolo M.E., Vilker A.S. (2010). Análisis de los determinantes de la variabilidad de los precios de las principales commodities exportadas por América Latina. XI Jornadas Nacionales y Latinoamericanas Actuariales. Facultad de Ciencias Económicas. Universidad de Buenos Aires.

Deaton, A. y Laroque, G. (1996). Competitive storage and commodity price dynamics. Journal of Political Economy, 104(5), 896-923.

Dornbusch, R. (1985). Policy and performance links between LDC debtors and industrial nations. Brookings Papers on Economic Activity, 1985(2), 303-368.

Dornbusch, R. (1976). Expectations and exchange rate dynamics. Journal of Political Economy, 84, 1161-1176.

Frankel, J. A. (1986). Expectations and commodity price dynamics: The overshooting model. American Journal of Agricultural Economics, 68(2), 344-348.

Frankel, J. A. (2006). The Effect of Monetary Policy on Real Commodity Prices. NBER Working Paper. No. 12713.

Henderson, B. J., Pearson, N. D. y Wang, L. (2012). New Evidence on the Financialization of Commodity Markets. Working Paper. George Washington University.

Hotelling, H. (1931). The economics of exhaustible resources. The Journal of Political Economy, 39(2), 137-175.

Hull, J. C. (2009). Options, Futures, and other Derivatives (7th edition). Pearson Prentice Hall.

Ivanov, V. y Kilian, L. (2005). A practitioner's guide to lag order selection for VAR impulse response analysis. Studies in Nonlinear Dynamics \& Econometrics, 9(1).

Kaplinsky, R. (2006). Revisiting the revisited terms of trade: Will China make a difference? World Development, 34(6), 981-995.

Kilian, L. y Park, C. (2009). The impact of oil price shocks on the US stock market. International Economic Review, 50(4), 1267-1287.

Le Clech, N. A. (2013). Determinantes del precio internacional de la soja. Atlantic Review of Economics, 2(1), 9-22. 
Maizels, A. (1994). Commodity market trends and instabilities: Policy options for developing countries (pp. 53-64). Geneva: UNCTAD Review.

Massot, J. (2008). Administración del riesgo económico y el boom de las commodities: el caso argentino. Impactos de la Crisis Financiera Internacional en la Argentina, 1, 15-29.

Nissanke M. (2010). Commodity markets and excess volatility: Sources and strategies to reduce adverse development impacts. Amsterdam, Common Fund for Commodities [consultado 29 May 2016]. Disponible en: http://common-fund.org/uploads/tx_cfc/CFC_report_Nissanke_Volatility_Development_Impact_2010_02.pdf

Silvennoinen, A. y Thorp, S. (2013). Financialization, crisis and commodity correlation dynamics. Journal of International Financial Markets, Institutions and Money, 24, 42-65.

Sims, C. (1980). Macroeconomics and reality. Econometrica., 48, 1-48.

Tang, K. y Xiong, W. (2012). Index investment and the financialization of commodities. Financial Analysts Journal, 68(5), 54-74.

United Nations (2009): United Nations Conference on Trade and Development, Geneva (2009): Trade and Development [consultado 29 May 2016]. Disponible en: http://www.unctad.org/en/docs/tdr2009_en.pdf

United Nations (2011). Price Formation in Financialized Commodity Markets: The Role of Information. New York and Geneva: United Nations.

Yang, J., Bessler, D. A. y Leatham, D. J. (2001). Asset storability and price discovery in commodity futures markets: a new look. Journal of Futures Markets, 21(3), 279-300. 